\title{
FOCUSED ACCELERATION OF COSMIC-RAY PARTICLES IN NON-UNIFORM MAGNETIC FIELDS
}

\author{
YuRi E. LITVINENKO ${ }^{1}$ AND R. SCHLICKEISER ${ }^{2}$ \\ ${ }^{1}$ Department of Mathematics, University of Waikato, P.B. 3105, Hamilton, New Zealand \\ ${ }^{2}$ Institüt für Theoretische Physik, Lehrstuhl IV: Weltraum- und Astrophysik, Ruhr-Universität Bochum, D-44780 Bochum, Germany \\ Received 2011 February 11; accepted 2011 April 4; published 2011 April 19
}

\begin{abstract}
The Fokker-Planck equation for cosmic-ray particles in a spatially varying guide magnetic field in a turbulent plasma is analyzed. An expression is derived for the mean rate of change of particle momentum, caused by the effect of adiabatic focusing in a non-uniform guide field. Results of an earlier diffusion-limit analysis are confirmed, and the physical picture is clarified by working directly with the Fokker-Planck equation. A distributed first-order Fermi acceleration mechanism is identified, which can be termed focused acceleration. If the forwardand backward-propagating waves have equal polarizations, focused acceleration operates when the net cross helicity of an Alfvénic slab turbulence is either negative in a diverging guide field or positive in a converging guide field. It is suggested that focused acceleration can contribute to the formation of the anomalous cosmic-ray spectrum at the heliospheric termination shock.
\end{abstract}

Key words: acceleration of particles - cosmic rays - turbulence

\section{INTRODUCTION}

One of the most important problems of modern astrophysics is to explain how cosmic-ray particles are accelerated to relativistic energies in powerful sources of nonthermal radiation. For a long time, diffusive first-order Fermi acceleration at shock fronts has been regarded as a prime candidate for particle acceleration in astrophysics (for reviews see Drury 1983; Blandford \& Eichler 1987). Modern TeV air-Cherenkov telescopes have indeed resolved the shock regions in supernova remnants and identified the shocks as strong emission regions of $\mathrm{TeV}$ photons generated by the accelerated particles (Hinton \& Hofmann 2009). Yet the recent observations of energetic anomalous cosmic-ray ions in the vicinity of the solar wind termination shock by the Voyager spacecraft indicated unexpectedly that the intensity of the anomalous cosmic rays did not peak at the shock (Stone et al. 2005, 2008; Decker et al. 2008), in sharp contrast to the prediction of diffusive shock acceleration theory. This disagreement motivates revisiting the problem of cosmic-ray acceleration in partially turbulent cosmic magnetic fields.

An important aspect, left out in the earlier work on cosmicray particle acceleration, is the presence of large-scale spatial gradients in the guide magnetic field. The gradients modify the mirror force that acts on the energetic particles, adding to the Lorentz force exerted by the guide magnetic field and the superimposed turbulent magnetic and electric fields from magnetohydrodynamic plasma fluctuations. Roelof (1969), Earl (1976), Kunstmann (1979), and Spangler \& Basart (1981) identified an effect of adiabatic focusing due to particle pitchangle scattering by the turbulent magnetic field in the presence of the mirror force. Adiabatic focusing has been shown to modify particle transport along the guide magnetic field and to give an additional spatial convective term in the equation for an evolving particle distribution.

Schlickeiser \& Shalchi (2008) and Schlickeiser (2009) recently argued that, similarly, the joint action of the mirror force and particle scattering by the turbulent electric field component also leads to an additional convective term in momentum space. The new convective term represents either first-order Fermi acceleration of particles, referred to as focused acceleration, or deceleration of particles, referred to as focused de- celeration, depending on the sign of the product $L H_{c}$. Here, $L^{-1}=-d \ln B_{0}(z) / d z$ denotes the focusing length of the guide magnetic field $B_{0}(z)$ and $H_{c}=\left(I^{+}-I^{-}\right) /\left(I^{+}+I^{-}\right) \in[-1,1]$ is the cross helicity of magnetohydrodynamic plasma waves, defined by the relative intensities of forward $\left(I^{+}\right)$and backward $\left(I^{-}\right)$propagating waves. It should be emphasized from the outset that degenerate $\left(H_{c} \rightarrow \pm 1\right)$ cross helicity values are expected in the upstream region of shock waves in space, because the upstream precursor cosmic-ray distribution function amplifies (damps) the forward (backward) propagating waves (Schlickeiser \& Shalchi 2008).

The purpose of this paper is twofold. First, we present an alternative derivation for the rate of focused acceleration/ deceleration, based on the Fokker-Planck cosmic-ray transport equation, clarifying the physical picture by a simple and direct method. Second, we suggest that the additional effect of focused acceleration/deceleration can contribute to the formation of anomalous cosmic-ray distributions observed near the heliospheric termination shock. Our analysis strongly suggests that the plasma microphysics of plasma wave generation, amplification, and damping is decisive for the efficient diffusive shock acceleration of cosmic-ray particles.

\section{COSMIC-RAY TRANSPORT IN NON-UNIFORM MAGNETIC FIELDS}

Transport of cosmic-ray particles in turbulent interstellar and interplanetary electromagnetic fields, superimposed on a largescale guide magnetic field, is typically described using the Fokker-Planck equation for the particle phase-space distribution function. Often the diffusion approximation is adopted, which is justified when the particle distribution is kept almost isotropic by a strong pitch-angle diffusion (Jokipii 1966; Hasselmann \& Wibberenz 1968; Kulsrud \& Pearce 1969; Earl 1974; Skilling 1975; Schlickeiser 1989).

The guide magnetic field is often non-uniform. The solar magnetic field in interplanetary space, for instance, can be described by the Parker (1958) spiral. Radio continuum surveys imply that large-scale spatial variations of the magnetic field perpendicular to the galactic plane are present in disk galaxies (e.g., Sofue et al. 1986). Spatially varying magnetic fields are 
believed to play a significant part in particle acceleration by shocks in the solar corona (e.g., Sandroos \& Vainio 2007). A feature of interest is the effect of adiabatic focusing in a spatially varying guide magnetic field, which leads to an additional convective term describing particle transport along the field. The resulting focused particle transport in interplanetary space has been repeatedly investigated (e.g., Kocharov et al. 1998; Bieber et al. 2002; Dröge et al. 2006; Sáiz et al. 2008).

Recently the diffusion approximation has been used systematically to describe the effects of a spatially varying guide magnetic field on the isotropic part of the cosmic-ray particle distribution in the weak focusing limit, when the focusing length $|L|$ is much greater than the scattering length $\lambda_{\|}$of cosmic-ray particles (Schlickeiser \& Shalchi 2008). As mentioned above, the combination of particle scattering by the turbulent electric field and the magnetic mirror force was shown to give rise to a new Fermi-type mechanism of particle acceleration when $H_{c} L<$ 0 . This new mechanism of focused acceleration has potentially important applications in several astrophysical situations (Schlickeiser \& Shalchi 2008; Schlickeiser 2009; Schlickeiser \& Jenko 2010), and so an independent calculation of the acceleration rate would be useful in verifying the original diffusion-limit analysis. Below we use the Fokker-Planck equation and derive an expression for the mean rate of change of particle momentum, caused by the adiabatic focusing term in a non-uniform magnetic guide field present in a turbulent plasma.

\section{FIRST-ORDER FERMI ACCELERATION IN A NON-UNIFORM GUIDE MAGNETIC FIELD}

We consider the Fokker-Planck equation that takes into account the effect of adiabatic focusing in a non-uniform magnetic guide field (Schlickeiser \& Jenko 2010):

$$
\begin{aligned}
\frac{\partial f_{0}}{\partial t} & +\mu v \frac{\partial f_{0}}{\partial z}+\frac{v}{2 L}\left(1-\mu^{2}\right) \frac{\partial f_{0}}{\partial \mu}=\frac{\partial}{\partial \mu}\left(D_{\mu \mu} \frac{\partial f_{0}}{\partial \mu}+D_{\mu p} \frac{\partial f_{0}}{\partial p}\right) \\
& +\frac{1}{p^{2}} \frac{\partial}{\partial p}\left(p^{2} D_{\mu p} \frac{\partial f_{0}}{\partial \mu}+p^{2} D_{p p} \frac{\partial f_{0}}{\partial p}\right) .
\end{aligned}
$$

Here, $f_{0}(z, p, \mu, t)$ is the gyrotropic particle phase-space density per unit of magnetic line length, which depends on the spatial coordinate $z$ along the guide magnetic field $B_{0}(z)$, the particle momentum $p$, the pitch-angle cosine $\mu$, and time $t$; $v$ is the particle speed. The focusing length $L$ is defined by $L^{-1}(z)=-d \ln B_{0}(z) / d z$.

In order not to obscure the essential points of our analysis of focused acceleration, we do not consider a particle source term, continuous and catastrophic momentum loss processes, or the dependence of $f_{0}$ on $x$ and $y$, so that particle drift effects are neglected on the left-hand side of Equation (1). Schlickeiser \& Jenko (2010) specifically addressed the effects of the curvature and gradient drifts in the kinetic equation (see their Equation (2.19)). Our approach should be valid as long as the spatial scales of the $x$ - and $y$-components of the magnetic field are large compared with that of the $z$-component of the field. For simplicity, we also assume $L=$ const on scales much larger than the cosmic-ray parallel scattering length (weak focusing limit) and adopt the partition scaling for the background gas density $n(z) \propto B_{0}^{2}(z)$, so that the Alfvén speed $v_{A}$ is constant. The transport equation (1) with gyrophase-averaged Fokker-Planck coefficients for the gyrotropic distribution function of energetic particles is an approximation that requires the particle Larmor radius, gyroperiod, and the ratio $v_{A} / v$ to be small expansion parameters (see Schlickeiser 2011 for a recent systematic derivation). In this limit, the drift terms that we neglect and the focusing term in Equation (1) are connected with the conservation of the particle magnetic moment in the guide field $B_{0}$ that is much stronger than the turbulent magnetic field component $\delta B$ (e.g., Roelof 1969; Fahr et al. 1977).

We begin our analysis of Equation (1) by rewriting it in terms of a gyrotropic differential particle number density $f(z, p, \mu, t)$. The functions $f_{0}$ and $f$ are related by

$$
f_{0}(z, p, \mu, t)=\frac{\exp (-z / L)}{2 \pi p^{2}} f(z, p, \mu, t),
$$

and the particle density $n(z, t)$ is expressed as

$$
n(z, t)=\int_{0}^{\infty} \int_{-1}^{1} f d \mu d p .
$$

On substituting Equation (2) into Equation (1) and rearranging terms, we get

$$
\begin{aligned}
\frac{\partial f}{\partial t}= & -\frac{\partial}{\partial z}(\mu v f)-\frac{\partial}{\partial \mu}\left[\frac{v}{2 L}\left(1-\mu^{2}\right) f+\frac{\partial D_{\mu \mu}}{\partial \mu} f\right. \\
& \left.+\frac{1}{p^{2}} \frac{\partial\left(p^{2} D_{\mu p}\right)}{\partial p} f\right]-\frac{\partial}{\partial p}\left[\frac{\partial D_{\mu p}}{\partial \mu} f+\frac{1}{p^{2}} \frac{\partial\left(p^{2} D_{p p}\right)}{\partial p} f\right] \\
& +\frac{\partial^{2}}{\partial \mu^{2}}\left(D_{\mu \mu} f\right)+\frac{\partial^{2}}{\partial \mu \partial p}\left(2 D_{\mu p} f\right)+\frac{\partial^{2}}{\partial p^{2}}\left(D_{p p} f\right) .
\end{aligned}
$$

We now use this form of the Fokker-Planck equation to derive equations for the mean values of the pitch-angle cosine

$$
\langle\mu\rangle=\left(\int_{-\infty}^{\infty} n d z\right)^{-1} \int_{-\infty}^{\infty} \int_{0}^{\infty} \int_{-1}^{1} \mu f d \mu d p d z
$$

and the particle momentum

$$
\langle p\rangle=\left(\int_{-\infty}^{\infty} n d z\right)^{-1} \int_{-\infty}^{\infty} \int_{0}^{\infty} \int_{-1}^{1} p f d \mu d p d z .
$$

On multiplying Equation (4) by either $\mu$ or $p$ and integrating with respect to $\mu, p$, and $z$, we obtain

$$
\begin{gathered}
\frac{d\langle\mu\rangle}{d t}=\left\langle\frac{v}{2 L}\left(1-\mu^{2}\right)+\frac{\partial D_{\mu \mu}}{\partial \mu}+\frac{1}{p^{2}} \frac{\partial\left(p^{2} D_{\mu p}\right)}{\partial p}\right\rangle, \\
\frac{d\langle p\rangle}{d t}=\left\langle\frac{\partial D_{\mu p}}{\partial \mu}+\frac{1}{p^{2}} \frac{\partial\left(p^{2} D_{p p}\right)}{\partial p}\right\rangle .
\end{gathered}
$$

To give a concrete example of practical interest, we now specialize to the case of isospectral undamped Alfvénic slab turbulence with constant magnetic and cross helicities that are independent of the wavenumber. The magnetic helicities $\sigma^{+}$and $\sigma^{-}$indicate the polarization states of the forwardand backward-propagating Alfvén waves. The cross helicity $H_{c}=\left(I^{+}-I^{-}\right) /\left(I^{+}+I^{-}\right)$specifies the relative intensities $I^{+}$and $I^{-}$of the forward- and backward-propagating waves. Schlickeiser (2002) summarized the corresponding transport parameters. The coefficients appearing in the Fokker-Planck equation (1) are as follows:

$$
D_{\mu \mu}=D_{0} N(\mu)
$$




$$
\begin{gathered}
D_{\mu p}=\epsilon p D_{0} M(\mu), \\
D_{p p}=\epsilon^{2} p^{2} D_{0} R(\mu), \\
D_{0}=\frac{\pi}{2}(s-1) v k_{\min }\left(k_{\min } R_{\mathrm{g}}\right)^{s-2}\left(\frac{\delta B}{B_{0}}\right)^{2}\left(1-\mu^{2}\right) .
\end{gathered}
$$

Here, $\epsilon=v_{A} / v$ where $v_{A}$ is the Alfvén speed, so that $\epsilon$ is typically a small parameter; $s$ is a power-law spectral index of the magnetic field correlation tensor above the minimum wavenumber $k_{\min }, R_{\mathrm{g}}$ is the particle gyroradius, and $\delta B$ is the fluctuating magnetic field amplitude. The functions $N(\mu)$ and $M(\mu)$ are defined by

$$
\begin{aligned}
& N(\mu)=\left(1+H_{c}\right)(1-\epsilon \mu)^{2}|\mu-\epsilon|^{s-1}\left\{\left(1+\sigma^{+}\right) H[Z(\epsilon-\mu)]\right. \\
& \left.+\left(1-\sigma^{+}\right) H[Z(\mu-\epsilon)]\right\}+\left(1-H_{c}\right)(1+\epsilon \mu)^{2}|\mu+\epsilon|^{s-1} \\
& \quad \times\left\{\left(1+\sigma^{-}\right) H[-Z(\epsilon+\mu)]+\left(1-\sigma^{-}\right) H[Z(\epsilon+\mu)]\right\},
\end{aligned}
$$

$$
\begin{aligned}
& M(\mu)=\left(1+H_{c}\right)(1-\epsilon \mu)|\mu-\epsilon|^{s-1}\left\{\left(1+\sigma^{+}\right) H[Z(\epsilon-\mu)]\right. \\
& \left.\quad+\left(1-\sigma^{+}\right) H[Z(\mu-\epsilon)]\right\}-\left(1-H_{c}\right)(1+\epsilon \mu)|\mu+\epsilon|^{s-1} \\
& \quad \times\left\{\left(1+\sigma^{-}\right) H[-Z(\epsilon+\mu)]+\left(1-\sigma^{-}\right) H[Z(\epsilon+\mu)]\right\} .
\end{aligned}
$$

Here, $H$ is the Heaviside step function and $Z=q /|q|$ where $q$ is the particle charge. An explicit expression for $R(\mu)$ will not be necessary since $D_{p p}$ is a higher-order term with respect to the small parameter $\epsilon$.

Next we make the key assumption that the scattering rate $D_{0}$ is large enough to ensure that the rate of change of the mean pitch-angle cosine $\langle\mu\rangle$ is at most a quantity of order $\epsilon$. Balancing the leading-order terms in Equations (7) and (8), we have

$$
\begin{gathered}
0=\left\langle\frac{v}{2 L}\left(1-\mu^{2}\right)+\frac{\partial D_{\mu \mu}}{\partial \mu}\right\rangle, \\
\frac{d\langle p\rangle}{d t}=\left\langle\frac{\partial D_{\mu p}}{\partial \mu}\right\rangle .
\end{gathered}
$$

To complete the derivation, we need to express $D_{\mu p}$ in terms of $D_{\mu \mu}$. The relationship is particularly simple if $\sigma^{+}=\sigma^{-}$. Then it follows from Equations (13) and (14) that

$$
D_{\mu p}=\epsilon p H_{c} D_{\mu \mu}+o(\epsilon) \text {. }
$$

On eliminating $D_{\mu p}$ from Equation (16), we have

$$
\frac{d\langle p\rangle}{d t}=\left\langle\epsilon p H_{c} \frac{\partial D_{\mu \mu}}{\partial \mu}\right\rangle \text {. }
$$

In the weak focusing limit of a large $L$, say in the parameter range

$$
\epsilon \ll \frac{\left(1-\mu^{2}\right) v}{D_{0} L} \ll 1,
$$

the particle distribution is almost isotropic. To a leading order in $L^{-1}$, we can use $\left\langle\mu^{2}\right\rangle=1 / 3$ and replace the mean of a product by the product of the means. Therefore, substituting Equation (15) into Equation (18) and remembering that $\epsilon v=$ $v_{A}$, we obtain

$$
\frac{1}{\langle p\rangle} \frac{d\langle p\rangle}{d t}=-\frac{v_{A} H_{c}}{3 L} .
$$

As long as $L<\infty$, the particles are losing momentum if $H_{c} L>0$ and gaining momentum if $H_{c} L<0$. The latter case corresponds to particle acceleration caused by the adiabatic focusing term in the Fokker-Planck equation-an effect first identified by Schlickeiser \& Shalchi (2008).

It is straightforward to generalize the argument to describe the case of unequal wave polarizations, $\sigma^{+} \neq \sigma^{-}$. When $Z=1$, for instance, Equations (13) and (14) give

$$
\frac{M}{N}=\frac{\left(1+H_{c}\right)\left(1-\sigma^{+}\right)-\left(1-H_{c}\right)\left(1-\sigma^{-}\right)}{\left(1+H_{c}\right)\left(1-\sigma^{+}\right)+\left(1-H_{c}\right)\left(1-\sigma^{-}\right)}+o(\epsilon)
$$

for $\mu>0$ and

$$
\frac{M}{N}=\frac{\left(1+H_{c}\right)\left(1+\sigma^{+}\right)-\left(1-H_{c}\right)\left(1+\sigma^{-}\right)}{\left(1+H_{c}\right)\left(1+\sigma^{+}\right)+\left(1-H_{c}\right)\left(1+\sigma^{-}\right)}+o(\epsilon)
$$

for $\mu<0$. Following the same steps as above, we get

$$
\frac{d\langle p\rangle}{d t}=\left\langle\epsilon p \frac{M}{N} \frac{\partial D_{\mu \mu}}{\partial \mu}\right\rangle,
$$

which is a generalization of Equation (18), and

$$
\frac{1}{\langle p\rangle} \frac{d\langle p\rangle}{d t}=-\frac{v_{A}}{3 L} \int_{-1}^{1} \frac{M}{2 N} d \mu,
$$

which is a generalization of Equation (20). The integral is easily evaluated by splitting the integration interval into the intervals $-1<\mu<0$ and $0<\mu<1$ and using Equations (21) and (22). After some algebra we finally arrive at

$$
\frac{1}{\langle p\rangle} \frac{d\langle p\rangle}{d t}=-\frac{v_{A} H}{3 L},
$$

where the function $H\left(H_{c}, \sigma^{+}, \sigma^{-}\right)$is defined by

$$
H=\frac{\left(1+H_{c}\right)^{2}\left[1-\left(\sigma^{+}\right)^{2}\right]-\left(1-H_{c}\right)^{2}\left[1-\left(\sigma^{-}\right)^{2}\right]}{\left(1+H_{c}\right)^{2}\left[1-\left(\sigma^{+}\right)^{2}\right]+\left(1-H_{c}\right)^{2}\left[1-\left(\sigma^{-}\right)^{2}\right]+2\left(1-H_{c}^{2}\right)\left(1-\sigma^{+} \sigma^{-}\right)} .
$$

The expression is identical to Equation (22) in Schlickeiser \& Shalchi (2008).

\section{FOCUSED ACCELERATION/DECELERATION NEAR THE TERMINATION SHOCK}

Schlickeiser (2009) argued that focused acceleration can operate in the interstellar medium, overcoming the Coulomb and ionization losses and accelerating cosmic-ray hadrons over 10 orders of magnitude in momentum. We suggest here that the mechanism of focused acceleration/deceleration may also contribute to the acceleration/deceleration of the anomalous cosmic rays at the heliospheric termination shock, because the upstream Parker field is condensed on the downstream side of the termination shock so that an inhomogeneous magnetic field structure is seen by galactic and anomalous cosmic rays.

Observations of energetic ions in the vicinity of the termination shock by the Voyager spacecraft have been analyzed using a focused transport model for shock acceleration (Le Roux et al. 2007; Le Roux \& Webb 2009). A puzzle remains, however, that the intensity of anomalous cosmic rays did not peak at the shock (Stone et al. 2005, 2008). One possible explanation is that stochastic acceleration of particles by Alfvén waves in the heliosheath contributes to the observed anomalous cosmic-ray 
spectrum (e.g., Ferreira et al. 2007). We propose that focused acceleration can be the specific acceleration mechanism. For $H_{c} L<0$, say due to an excess of the backward-propagating Alfvén waves in a diverging upstream guide magnetic field, the efficiency of focused acceleration should exceed that of the standard diffusive shock acceleration for sufficiently small Alfvén Mach numbers of the shock (Schlickeiser \& Shalchi 2008). Moreover, focused acceleration operates in the whole upstream region, explaining why the intensity of anomalous cosmic rays did not peak at the shock position.

Non-zero values of the upstream cross helicity should be expected because the upstream precursor cosmic-ray distribution function amplifies (damps) the forward- (backward-) propagating waves (Schlickeiser \& Shalchi 2008). Depending on the adopted spatial boundary conditions on the intensities of the waves, this effect leads to either $H_{c} \rightarrow 1$ or $H_{c} \rightarrow-1$. Schlickeiser \& Shalchi (2008) assumed that the forward- and backward-propagating waves have equal intensities far away from the shock at $x=0$, that is $H_{c}(x=\infty)=0$. This boundary condition gives rise to $H_{c}<0$ in the whole upstream region, which in a diverging guide magnetic field leads to focused acceleration in the upstream region. Alternatively, if we assume that the forward- and backward-propagating waves have equal intensities at the location of the shock, $H_{c}(x=0)=0$, we find that this boundary condition gives rise to $H_{c}>0$ in the whole upstream region. In a diverging guide magnetic field this leads to focused deceleration of cosmic-ray particles in the upstream region, with a loss rate overcoming the standard diffusive shock acceleration for sufficiently small Alfvén Mach numbers of the shock. In this case, cosmic-ray particles are not accelerated in the shock region. In a converging upstream guide magnetic field (negative focusing length $L<0$ ), focusing has the opposite effect: $H_{c}>0$ upstream then implies additional effective focused acceleration upstream, whereas $H_{c}<0$ upstream leads to significant focused deceleration of particles upstream.

Our analysis indicates that the plasma microphysics of plasma wave generation, amplification, and damping near shocks is decisive for the efficient first-order Fermi acceleration of cosmicray particles in diverging or converging guide magnetic fields. We suggest that the validity of the new acceleration mechanism could be tested by searching for a correlation between the accelerated particle flux, the cross helicity of the plasma wave fields, and the sign of the focusing length of the guide magnetic field. Of course, the field inhomogeneities in the heliosphere can be more complicated than the simple case of isospectral undamped Alfvénic slab turbulence with constant magnetic and cross helicities adopted here for illustrating the appearance and properties of the new focused acceleration/deceleration effect. Although various complications are likely to arise in a more detailed quantitative analysis, we expect that the basic focusing effect also occurs in more complex geometries, since the magnetic and electric field inhomogeneities in the solar wind rest frame, which scatter cosmic-ray particles in pitch angle and momentum, should generally result in a non-zero cross helicity and thus lead to directional anisotropy.

\section{DISCUSSION}

Our analysis shows that the mechanism of focused acceleration can accelerate charged particles in a turbulent plasma with a large-scale spatially varying magnetic field. For instance, if the forward- and backward-propagating waves have equal polarizations, $\sigma^{+}=\sigma^{-}$, the particles are accelerated when the net cross helicity of Alfvénic turbulence $H_{c}$ and the focusing length
$L$ of the guide magnetic field $B_{0}(z) \sim \exp (-z / L)$ satisfy the requirement $H_{c} L<0$, or in other words when the cross helicity $H_{c}$ is either negative in a diverging guide field or positive in a converging guide field.

To derive a formula for the acceleration rate, we assumed that pitch-angle scattering is strong enough to balance adiabatic focusing in Equation (15), resulting in a small mean pitchangle cosine. Although the diffusion approximation is based on a similar idea, our approach here is more general and has the advantage that we work with the original Fokker-Planck equation. In contrast to the diffusion approximation, a formula for the anisotropic part of the particle distribution function is not used in our calculation. Our method leads directly to an expression for the rate of change of the mean particle momentum and identifies the physical conditions under which particle acceleration can occur. On the other hand, the diffusion approximation leads to a general diffusion-convection transport equation that can be used systematically to investigate various transport effects in phase space. To sum up, the more general analysis of this paper provides an independent confirmation of the diffusion-limit calculation by Schlickeiser \& Shalchi (2008) and puts the new acceleration mechanism on a firmer footing.

We also suggest here that focused acceleration/deceleration may significantly contribute to the acceleration/deceleration of the anomalous cosmic rays at the heliospheric termination shock. The validity of the new acceleration/deceleration mechanism could be assessed by searching for a correlation between the accelerated particle flux, the cross helicity of the plasma wave fields, and the sign of the focusing length of the guide magnetic field. Hence, our analysis suggests a testable explanation of the current observational puzzle (Stone et al. 2005, 2008; Decker et al. 2008).

Y.L. was supported by NASA grant NNX08AG44G and by a research fellowship from the Alexander von Humboldt Foundation. R.S. acknowledges support by the Deutsche Forschungsgemeinschaft (grant Schl 201/23-1).

\section{REFERENCES}

Bieber, J. W., et al. 2002, ApJ, 567, 622

Blandford, R. D., \& Eichler, D. 1987, Phys. Rep., 154, 1

Decker, R. B., Krimigis, S. M., Roelof, E. C., Hill, M. E., Armstrong, T. P., Gloeckler, G., Hamilton, D. C., \& Lanzerotti, L. J. 2008, Nature, 454, 67 Dröge, W., Kartavykh, Y. Y., Klecker, B., \& Mason, G. M. 2006, ApJ, 645, 1516 Drury, L. O. C. 1983, Rep. Progr. Phys., 46, 973

Earl, J. A. 1974, ApJ, 193, 231

Earl, J. A. 1976, ApJ, 205, 900

Fahr, H. J., Bird, M. K., \& Ripken, H. W. 1977, A\&A, 58, 339

Ferreira, S. E. S., Potgieter, M. S., \& Scherer, K. 2007, J. Geophys. Res., 112, A11101

Hasselmann, K., \& Wibberenz, G. 1968, Z. Geophys., 34, 353

Hinton, J. A., \& Hofmann, W. 2009, ARA\&A, 47, 523

Jokipii, J. R. 1966, ApJ, 146, 480

Kocharov, L., Vainio, R., Kovaltsov, G. A., \& Torsti, J. 1998, Sol. Phys., 182, 195

Kulsrud, R., \& Pearce, W. P. 1969, ApJ, 156, 445

Kunstmann, J. 1979, ApJ, 229, 812

Le Roux, J. A., \& Webb, G. M. 2009, ApJ, 693, 534

Le Roux, J. A., Webb, G. M., Florinski, V., \& Zank, G. P. 2007, ApJ, 662, 350 Parker, E. N. 1958, ApJ, 128, 664

Roelof, E. C. 1969, in Lectures in High Energy Astrophysics, ed. H. Ögelman \& J. R. Wayland (NASA SP-199; Washington, DC: NASA), 111

Sáiz, A., Ruffolo, D., Bieber, J. W., Evenson, P., \& Pyle, R. 2008, ApJ, 672, 650 Sandroos, A., \& Vainio, R. 2007, A\&A, 455, 685

Schlickeiser, R. 1989, ApJ, 336, 243

Schlickeiser, R. 2002, Cosmic Ray Astrophysics (Heidelberg: Springer), chap. 13

Schlickeiser, R. 2009, Mod. Phys. Lett. A, 24, 1461 
Schlickeiser, R. 2011, ApJ, 732, 96

Schlickeiser, R., \& Jenko, F. 2010, J. Plasma Phys., 76, 317

Schlickeiser, R., \& Shalchi, A. 2008, ApJ, 686, 292

Skilling, J. 1975, MNRAS, 172, 557

Sofue, Y., Fujimoto, M., \& Wielebinski, R. 1986, ARA\&A, 24, 459
Spangler, S. R., \& Basart, J. P. 1981, ApJ, 243, 1103

Stone, E. C., Cummings, A. C., McDonald, F. B., Heikkila, B. C., Lal, N., \& Webber, W. R. 2005, Science, 309, 2017

Stone, E. C., Cummings, A. C., McDonald, F. B., Heikkila, B. C., Lal, N., \& Webber, W. R. 2008, Nature, 454, 73 